\title{
MONOIDS OVER WHICH ALL WEAKLY FLAT ACTS ARE FLAT
}

\author{
by SYDNEY BULMAN-FLEMING and KENNETH McDOWELL* \\ (Received 17th January 1989)
}

\begin{abstract}
If $R$ is a ring with identity and $M$ is a left $R$-module then it is well known that the following statements are equivalent:

(1) $M$ is flat.

(2) The functor $-\otimes M$ preserves embeddings of right ideals into $R$.

This paper investigates situations in which the analogous statements are equivalent in the context of $S$-sets over a monoid $S$ :
\end{abstract}

1980 Mathematics subject classification (1985 Revision): Primary 20M10; Secondary $20 \mathrm{M} 50$.

\section{Introduction}

If $R$ is a ring with identity and $M$ is a left $R$-module then it is well known that the following statements are equivalent $[1]$ :

(1) $M$ is flat.

(2) The functor $-\otimes M$ preserves embeddings of (finitely generated) right ideals into $R$.

In this paper we investigate the equivalence of the analogous statements in the context of $S$-sets over a monoid $S$.

Let $S$ be monoid. If $A$ is a right $S$-set and $B$ is a left $S$-set the tensor product $A \otimes B$ has been studied extensively (see, for example, [7] or [2]). We will use the following terminology, which is consistent with that appearing in [14], [10] and [8].

Definition 1.1. Let $S$ be a monoid and $B$ be a left $S$-set. $B$ is called flat if the functor $-\otimes B$ preserves embeddings of right $S$-sets. If this functor preserves embeddings of [principal] right ideals of $S$ into $S$ (considered as a right $S$-set) then $B$ is called [principally] weakly flat.

Let $A$ be a right $S$-set, $B$ be a left $S$-set, $a, a^{\prime} \in A$ and $b, b^{\prime} \in B$. It is shown in [2] that

* Regretfully the second author died in September 1988. Research supported by Natural Sciences and Engineering Research Council of Canada grants A4494 and A9241. 
$a \otimes b=a^{\prime} \otimes b^{\prime}$ in $A \otimes B$ if and only if there exist $a_{1}, \ldots, a_{n} \in A, b_{2}, \ldots, b_{n} \in B$ and $s_{1}, t_{1}, \ldots, s_{n}, t_{n} \in S$ such that

$$
\begin{array}{rlrl}
a & =a_{1} s_{1} & \\
a_{1} t_{1} & =a_{2} s_{2} & s_{1} b=t_{1} b_{2} \\
a_{2} t_{2} & =a_{3} s_{3} & s_{2} b_{2}=t_{2} b_{3} \\
\vdots & \vdots \\
a_{n} t_{n} & =a^{\prime} & s_{n} b_{n}=t_{n} b^{\prime}
\end{array}
$$

This system of equalities is called a scheme over $A$ and $B$ of length $n$ joining $(a, b)$ to $\left(a^{\prime}, b^{\prime}\right)$. Thus $B$ is flat if and only if, for every right $S$-set $A$, and every $a, a^{\prime} \in A, b, b^{\prime} \in B$ such that there exists a scheme over $A$ and $B$ joining $(a, b)$ to $\left(a^{\prime}, b^{\prime}\right)$, there exists a scheme (of possibly different length) over $a S \cup a^{\prime} S$ and $B$ joining $(a, b)$ to $\left(a^{\prime}, b^{\prime}\right)$. Furthermore, using the fact that $S \otimes B \cong B$, it is easy to see that $B$ is weakly flat if and only if for every $x, y \in S$ and $b, b^{\prime} \in B, x b=y b^{\prime}$ implies there exist $x_{1}, \ldots, x_{n} \in\{x, y\}$, $b_{2}, \ldots, b_{n} \in B$ and $u_{1}, v_{1}, \ldots, u_{n}, v_{n}$ in $S$ such that

$$
\begin{array}{cc}
x=x_{1} u_{1} & \\
x_{1} v_{1}=x_{2} u_{2} & u_{1} b=v_{1} b_{2} \\
\vdots & \vdots \\
x_{n} v_{n}=y & u_{n} b_{n}=v_{n} b^{\prime} .
\end{array}
$$

A similar description in which $x=y$ characterizes when $B$ is principally weakly flat.

A monoid $S$ is called [weakly] [left, right] absolutely flat if all its [left, right] $S$-sets are [weakly] flat.

Let $E(S)$ denote the set of idempotents of a monoid $S$. If $x \in S$ the principal right ideal $x S$ is projective if and only if there exists $u \in E(S)$ such that $x u=x$, and whenever $x s=x t$ $(s, t \in S$ ) it follows that $u s=u t$ (see [12]). $S$ is called right $P P$ [9] if all of its principal right ideals are projective. The class of these monoids, which we shall consider in Section 2 , is extensive in that it contains all regular and all left cancellative monoids.

To conclude this introduction we provide a description of weak flatness which will be used throughout the paper. This generalizes Lemma 2 of [10].

Proposition 1.2. A left $S$-set $B$ is weakly flat if and only if

(i) $B$ is principally weakly flat, and

(ii) for all right ideals $I$ and $J$ of $S, I B \cap J B=(I \cap J) B$. 
Remark. In the sequel we use the following, equivalent, formulation of (ii): for all $x, y \in S$ and $b, b^{\prime} \in B$, if $x b=y b^{\prime}$ then there exist $b^{\prime \prime} \in B$ and $z \in x S \cap y S$ such that $x b=y b^{\prime}=z b^{\prime \prime}$.

Proof of 1.2. Assume first that $B$ is weakly flat. Then condition (i) follows immediately. To obtain (ii) suppose $x, y \in S$ and $b, b^{\prime} \in B$ are such that $x b=y b^{\prime}$. Because $B$ is weakly flat, there exists a scheme

$$
\begin{array}{cc}
x & =x_{1} u_{1} \\
x_{1} v_{1}=x_{2} u_{2} & u_{1} b=v_{1} b_{2} \\
\vdots & \vdots \\
x_{n} v_{n}=y & u_{n} b_{n}=v_{n} b^{\prime}
\end{array}
$$

where $x_{i} \in\{x, y\}$ for $1 \leqq i \leqq n, u_{1}, v_{1}, \ldots, u_{n}, v_{n} \in S$, and $b_{2}, \ldots, b_{n} \in B$. Let $z_{1}=x$ and for $2 \leqq i \leqq n+1$ let $z_{i}=x_{i-1} v_{i-1}$. There exists some $k \in\{1, \ldots, n+1\}$ such that $z_{k} \in x S \cap y S$. Since it is easy to check that $x b=y b^{\prime}=z_{1} b=z_{n+1} b^{\prime}=z_{k} b_{k}$ for $2 \leqq k \leqq n$, (ii) holds.

Now assume conditions (i) and (ii) and suppose $x \otimes b=y \otimes b^{\prime}$ in $S \otimes B$ (i.e. $x b=y b^{\prime}$ in $B$ ) for some $b, b^{\prime} \in B$ and $x, y \in S$. We must show $x \otimes b=y \otimes b^{\prime}$ in $(x S \cup y S)^{\prime} \otimes B$. By (ii) there exists $z \in x S \cap y S$ and $b^{\prime \prime} \in B$ such that $x b=y b^{\prime}=z b^{\prime \prime}$. Because $x \otimes b=z \otimes b^{\prime \prime}$ in $S \otimes B$ this equality also holds in $x S \otimes B$ by (i). Similarly, $y \otimes b^{\prime}=z \otimes b^{\prime \prime}$ in $y S \otimes B$. Hence, $x \otimes b=y \otimes b^{\prime}$ in $(x S \cup y S) \otimes B$.

\section{Right $P P$ monoids}

Lemma 2.1. Let $S$ be a right $P P$ monoid. $A$ left $S$-set $B$ is principally weakly flat if and only if, for every $b, b^{\prime} \in B$ and $x \in S, x b=x b^{\prime}$ implies there exists $u \in E(S)$ such that $x u=x$ and $u b=u b^{\prime}$.

Proof. Suppose $B$ is principally weakly flat, $b, b^{\prime} \in B, x \in S$, and $x b=x b^{\prime}$. Then there exist $u_{1}, \ldots, u_{n}, v_{1}, \ldots, v_{n} \in S$ and $b_{2}, \ldots, b_{n} \in B$ such that

$$
\begin{array}{cc}
x=x u_{1} & \\
x v_{1}=x u_{2} & u_{1} b=v_{1} b_{2} \\
\vdots & \vdots \\
x v_{n}=x & u_{n} b_{n}=v_{n} b^{\prime} .
\end{array}
$$

Since $S$ is right $P P$ there exists $u \in E(S)$ such that $x u=x$ and, whenever $x s=x t(s, t \in S)$, it 
follows that $u s=u t$. Therefore, $u=u u_{1}, u v_{i}=u u_{i+1}(1 \leqq i \leqq n-1), u v_{n}=u$, and so $u b=$ $u u_{1} b=u v_{1} b_{2}=u u_{2} b_{2}=\cdots=u u_{n} b_{n}=u v_{n} b^{\prime}=u b^{\prime}$ as required.

Now suppose the condition holds for $B$ and $x \otimes b=x \otimes b^{\prime}$ in $S \otimes B\left(x \in S, b, b^{\prime} \in B\right)$. This means $x b=x b^{\prime}$ in $B$ and so, in $x S \otimes B, x \otimes b=x u \otimes b=x \otimes u b=x \otimes u b^{\prime}=$ $x u \otimes b^{\prime}=x \otimes b^{\prime}$, and thus $B$ is principally weakly flat.

Corollary 2.2. If $S$ is a left cancellative monoid, then a left $S$-set $B$ is principally weakly flat if and only if it is torsion-free (see [13]).

If $\omega$ denotes the additive monoid of non-negative integers then the ideal $(\omega \times \omega) \backslash$ $\{(0,0)\}$ of the cancellative monoid $S=\omega \times \omega$ is principally weakly flat. However, this ideal is not weakly flat (see Proposition 2.5 , for example).

It is apparent from Lemma 2.1 that if $S$ is a regular monoid, every (left) $S$-set is principally weakly flat. (The converse is also true [10].) The condition in the following proposition appears in [8].

Proposition 2.3. Let $S$ be a regular monoid. Then a left $S$-set $B$ is weakly flat if and only if, for every $b \in B$ and $x, y \in S$, if $x b=y b$ then there exists $z \in x S \cap y S$ such that $x b=y b=z b$.

Proof. Suppose $B$ is weakly flat and $x b=y b$. Then by (ii) of Proposition 1.2 there exist $z_{1} \in x S \cap y S$ and $b^{\prime \prime} \in B$ such that $x b=y b=z_{1} b^{\prime \prime}$. If $z_{1}^{\prime} \in S$ satisfies $z_{1} z_{1}^{\prime} z_{1}=z_{1}$, then $z=z_{1} z_{1}^{\prime} x$ belongs to $x S \cap y S$ and satisfies $x b=y b=z b$.

Conversely suppose $B$ satisfies the stated condition. Since we already know $B$ is principally weakly flat, we show that (ii) of Proposition 1.2 holds. Suppose $x b=y b^{\prime}$, where $x, y \in S$ and $b, b^{\prime} \in B$. Let $x b=y b^{\prime}=b^{\prime \prime}$ and let $x^{\prime}, y^{\prime} \in S$ satisfy $x x^{\prime} x=x$ and $y y^{\prime} y=y$. Note that $b^{\prime \prime}=x x^{\prime} b^{\prime \prime}=y y^{\prime} b^{\prime \prime}$. Using the condition, this element is equal to $z b^{\prime \prime}$ for some $z \in x x^{\prime} S \cap y y^{\prime} S=x S \cap y S$, and the proof is complete.

The following result of V. Fleischer [8] is now easily obtained. For $x, y \in S$, $\theta_{L}(x, y)\left[\theta_{R}(x, y)\right]$ will denote the smallest left [right] congruence on $S$ containing $(x, y)$.

Corollary 2.4. For any monoid $S$ the following statements are equivalent:

(i) $S$ is weakly left absolutely flat.

(ii) $S$ is regular and $S$ satisfies

$(L)$ for all $x, y \in S$ there exists $z \in x S \cap y S$ such that $(z, x) \in \theta_{L}(x, y)$.

Proposition 2.5. Let $S$ be a right $P P$ monoid and let $B$ be a left $S$-set. Then $B$ is weakly flat if and only if, for all $x, y \in S$ and $b, b^{\prime} \in B$, if $x b=y b^{\prime}$ then there exist $b^{\prime \prime} \in B$, $x_{1}, y_{1} \in S$, and $u, v \in E(S)$ such that $x u=x, y v=y, u b=x_{1} b^{\prime \prime}, v b^{\prime}=y_{1} b^{\prime \prime}$ and $x x_{1}=y y_{1}$.

Proof. Suppose $B$ is weakly flat and assume $x b=y b^{\prime}$. By (ii) of Proposition 1.2 there 
exist $b^{\prime \prime} \in B$ and $z=x s=y t \in x S \cap y S$ such that $x b=y b^{\prime}=z b^{\prime \prime}$. From $x b=x\left(s b^{\prime \prime}\right)$ it follows from Lemma 2.1 that there exists $u \in E(S)$ such that $x u=x$ and $u b=u s b^{\prime \prime}$; similarly there exists $v \in E(S)$ such that $y v=y$ and $v b^{\prime}=v t b^{\prime \prime}$. We may now take $x_{1}=u s$ and $y_{1}=v t$.

Now assume the condition holds and suppose $x \otimes b=y \otimes b^{\prime}$ in $S \otimes B$ (i.e. $x b=y b^{\prime}$ in $B)$. Then $b^{\prime \prime} \in B, x_{1}, y_{1} \in S$, and $u, v \in E(S)$ exist as described above, and in $(x S \cup y S) \otimes B$ we calculate $x \otimes b=x u \otimes b=x \otimes u b=x \otimes x_{1} b^{\prime \prime}=x x_{1} \otimes b^{\prime \prime}=y y_{1} \otimes b^{\prime \prime}=y \otimes b^{\prime}$. Therefore $B$ is weakly flat.

In [8] V. Fleischer proved that, for any monoid $S$, if all left and right $S$-sets are weakly flat, then in fact they are all flat. Our paper [5] gives an alternative proof of this result. Using similar ideas we obtain the following:

Theorem 2.6. Let $S$ be any monoid. If

(i) $S$ is right $P P$, and

(ii) $S$ satisfies $\left(R^{\prime}\right)$ : for all $u, v \in E(S)$ there exists $z \in S u \cap S v$ such that $(z, u) \in \theta_{R}(u, v)$ then every weakly flat left $S$-set is flat.

Proof. Let $B$ denote any weakly flat left $S$-set, and choose an arbitrary right $S$-set, $A$. Suppose $a, a^{\prime} \in A$ and $b, b^{\prime} \in B$ are such that $a \otimes b=a^{\prime} \otimes b^{\prime}$ in $A \otimes B$. Then there exist $a_{1}, \ldots, a_{n} \in A, b_{2}, \ldots, b_{n} \in B, s_{1}, t_{1}, \ldots, s_{n}, t_{n} \in S$ such that

$$
\begin{array}{rlrl}
a & =a_{1} s_{1} & \\
a_{1} t_{1} & =a_{2} s_{2} & s_{1} b & =t_{1} b_{2} \\
& \vdots & \vdots \\
a_{i} t_{i} & =a_{i+1} s_{i+1} & s_{i} b_{i}=t_{i} b_{i+1} \\
& \vdots & \vdots \\
a_{n} t_{n} & =a^{\prime} & s_{n} b_{n}=t_{n} b^{\prime} .
\end{array}
$$

By Lemma 2.2 of [2] we must show there exists a replacement scheme over $a S \cup a^{\prime} S$ and $B$ joining $(a, b)$ to $\left(a^{\prime}, b^{\prime}\right)$. By Proposition 2.5 , for each $i \in\{1, \ldots, n\}$ there exist $b_{i}^{\prime \prime} \in B$, $x_{i}, y_{i} \in S$, and $u_{i}, v_{i} \in E(S)$ such that

$$
\begin{gathered}
s_{i} u_{i}=s_{i}, t_{i} v_{i}=t_{i} \\
u_{i} b_{i}=x_{i} b_{i}^{\prime \prime}, v_{i} b_{i+1}=y_{i} b_{i}^{\prime \prime} \\
s_{i} x_{i}=t_{i} y_{i} .
\end{gathered}
$$


(In (2) we define $b_{1}=b$ and $b_{n+1}=b^{\prime}$.) By $\left(R^{\prime}\right)$ there exist $q_{i}, r_{i} \in S(1 \leqq i \leqq n-1)$ such that

$$
q_{i} v_{i}=r_{i} u_{i+1}
$$

and $\left(r_{i} u_{i+1}, u_{i+1}\right) \in \theta_{R}\left(u_{i+1}, v_{i}\right)$. Because the relation $\rho_{i}=\left\{(s, t) \in S \times S \mid a_{i+1} s_{i+1} s=\right.$ $\left.a_{i+1} s_{i+1} t\right\}$ is a right congruence on $S$ containing $\left(u_{i+1}, v_{i}\right)$, we obtain

$$
a_{i+1} s_{i+1} r_{i} u_{i+1}=a_{i+1} s_{i+1}
$$

for $i \in\{1, \ldots, n-1\}$. We now proceed by induction on $n$.

If $n=1$ it is easy to see that (using (1) to (3))

$$
\begin{aligned}
a & =a u_{1} & \\
a x_{1} & =a^{\prime} y_{1} & u_{1} b=x_{1} b_{1}^{\prime \prime} \\
a^{\prime} v_{1} & =a^{\prime} & y_{1} b_{1}^{\prime \prime}=v_{1} b^{\prime}
\end{aligned}
$$

is a suitable replacement scheme. Now consider a scheme $(\Sigma)$ as above, where $n \geqq 2$. We first verify that

$$
\begin{aligned}
a & =a u_{1} \\
a x_{1} & =\left(a_{2} s_{2}\right) q_{1} v_{1} y_{1} \\
& \\
\left(a_{2} s_{2}\right) r_{1} u_{2} x_{2}=\left(a_{3} s_{3}\right) q_{2} v_{2} y_{2} & u_{1} b=x_{1} b_{1}^{\prime \prime} \\
\vdots & q_{1} v_{1} y_{1} b_{1}^{\prime \prime}=r_{1} u_{2} x_{2} b_{2}^{\prime \prime} \\
\left(a_{i} s_{i}\right) r_{i-1} u_{i} x_{i}=\left(a_{i+1} s_{i+1}\right) q_{i} v_{i} y_{i} & q_{i-1} v_{i-1} y_{i-1} b_{i-1}^{\prime \prime}=r_{i-1} u_{i} x_{i} b_{i}^{\prime \prime} \\
\vdots & \vdots \\
\left(a_{n} s_{n}\right) r_{n-1} u_{n} x_{n}=a^{\prime} y_{n} & q_{n-1} v_{n-1} y_{n-1} b_{n-1}^{\prime \prime}=r_{n-1} u_{n} x_{n} b_{n}^{\prime \prime} \\
\hline a^{\prime} v_{n}=a^{\prime} & y_{n} b_{n}^{\prime \prime}=v_{n} b^{\prime}
\end{aligned}
$$

is a scheme joining $(a, b)$ to $\left(a^{\prime}, b^{\prime}\right)$. The reader may verify that the equalities above hold. For example,

$$
\begin{aligned}
\left(a_{i} s_{i}\right) r_{i-1} u_{i} x_{i} & =a_{i} s_{i} x_{i} & & (\text { by }(5)) \\
& =a_{i} t_{i} y_{i} & & (\text { by }(3)) \\
& =a_{i+1} s_{i+1} y_{i} & & (\text { from }(\Sigma)) \\
& =a_{i+1} s_{i+1} r_{i} u_{i+1} y_{i} & & (\text { by }(5)) \\
& =\left(a_{i+1} s_{i+1}\right) q_{i} v_{i} y_{i} & & \text { (by }(4))
\end{aligned}
$$


and

$$
\begin{aligned}
q_{i-1} v_{i-1} y_{i-1} b_{i-1}^{\prime \prime} & =q_{i-1} v_{i-1}^{2} b_{i} & & (\text { by }(2)) \\
& =q_{i-1} v_{i-1} b_{i} & & \left(\text { since } v_{i-1} \in E(S)\right) \\
& =r_{i-1} u_{i} b_{i} & & (\text { by }(4)) \\
& =r_{i-1} u_{i}^{2} b_{i} & & \left(\text { since } u_{i} \in E(S)\right) \\
& =r_{i-1} u_{i} x_{i} b_{i}^{\prime \prime} & & (\text { by }(2)) .
\end{aligned}
$$

The proof is now completed as follows: the outlined subscheme above has length $n-1$ and so, using the inductive hypothesis, $a x_{1} \otimes b_{1}^{\prime \prime}=a^{\prime} y_{n} \otimes b_{n}^{\prime \prime}$ in $\left(a x_{1} S \cup a^{\prime} y_{n} S\right) \otimes B$, hence also in $\left(a S \cup a^{\prime} S\right) \otimes B$. But using the initial and final portions of the scheme above it is immediate that $a \otimes b=a x_{1} \otimes b_{1}^{\prime \prime}$ and $a^{\prime} y_{n} \otimes b_{n}^{\prime \prime}=a^{\prime} \otimes b^{\prime}$, also in $\left(a S \cup a^{\prime} S\right) \otimes B$. It follows that $a \otimes b=a^{\prime} \otimes b^{\prime}$ in $\left(a S \cup a^{\prime} S\right) \otimes B$, as required.

Corollary 2.7. Let $S$ be a monoid. Each of the following conditions implies every weakly flat $S$-set is flat.

(i) $S$ is weakly right absolutely flat.

(ii) $S$ is right $P P$ and its idempotents form a left regular band.

\section{Proof.}

(i) $S$ is regular and satisfies $(R)$, the dual of $(L)$, by Corollary 2.4. Hence, $S$ satisfies the hypotheses of Theorem 2.6.

(ii) $E(S)$ satisfies the identity $x y x=x y$. To verify $\left(R^{\prime}\right)$ suppose $u, v \in E(S)$. Then $z=v u v=v u \in S u \cap S v$ and $(z, u) \in \theta_{R}(u, v)$.

The reader should note that the following monoids satisfy (ii) of Corollary 2.7 :

(a) commutative $P P$ monoids (characterized in [12]);

(b) right $P P$ monoids with central idempotents (characterized in [9]) (This includes all left cancellative monoids, for example. For more information see [4].)

(c) $S^{1}$ where $S$ is any left generalized inverse semigroup (see [15]).

Corollary 2.8 ([8]). If a monoid $S$ is weakly absolutely flat then $S$ is absolutely flat.

The obvious one-sided analogue of Corollary 2.8 is not true. We consider the situation for bands, first noting the following:

Proposition 2.9. Let $S$ be an idempotent monoid. Then $S$ is weakly left absolutely flat if and only if $S$ is right regular. 
Proof. Suppose first that $S$ is a right regular band. By Corollary 2.4 we need only verify that $S$ satisfies $(L)$. Let $x, y \in S$. Then $z=x y x=y x \in x S \cap y S$ and $(z, x) \in \theta_{L}(x, y)$ (as in the proof of Corollary $2.7($ iii)).

Now assume $S$ is weakly left absolutely flat, and let $S=\cup_{\gamma \in \Gamma} S_{\gamma}$ where $\Gamma$ is a semilattice and each $S_{\gamma}$ is a rectangular band. We need to show that each $S_{\gamma}$ is a right zero band, so let $x, y \in S_{y}(\gamma \in \Gamma)$. By $(L)$ there exists $z=x z=y z \in x S \cap y S$ such that $(z, x) \in \theta_{L}(x, y)$, and thus there exist $u_{1}, \ldots, u_{n}, x_{1}, y_{1}, \ldots, x_{n}, y_{n} \in S$ with $\left\{x_{i}, y_{i}\right\}=\{x, y\}$ $(1 \leqq i \leqq n)$ for which

$$
\begin{aligned}
x & =u_{1} x_{1} \\
u_{1} y_{1} & =u_{2} x_{2} \\
\vdots & \\
u_{n} y_{n} & =z .
\end{aligned}
$$

It is easily seen that each of these $n+1$ elements and in particular, $z$, belongs to $S_{\gamma}$. Thus $x y=x y z y=x z y=y z y=y$ as required.

Example 2.10. Let $S$ be any right normal band which does not have constant structure maps. Then $S^{1}$ is weakly left absolutely flat, by the preceding proposition, but not left absolutely flat (see [3, Proposition 2.3]). However, note that by the dual of Corollary $2.7(\mathrm{ii})$, every weakly flat right $S^{1}$-set is flat.

\section{Other situations}

In this section we examine further situations in which weakly flat $S$-sets are flat. First we consider some technical matters.

Lemma 3.1. Suppose $S$ is a monoid, $A$ is a right $S$-set and $B$ is a left $S$-set which is principally weakly flat. Assume $a, a_{1} \in A, b, b_{1} \in B, s_{1} \in S$ are such that $a=a_{1} s_{1}$ and $s_{1} b=s_{1} b_{1}$. Then $a \otimes b=a \otimes b_{1}$ in $a S \otimes B$.

Proof. Since $s_{1} b=s_{1} b_{1}, s_{1} \otimes b=s_{1} \otimes b_{1}$ in $s_{1} S \otimes B$ because $B$ is principally weakly flat. Therefore $u_{1}, v_{1} \ldots, u_{n}, v_{n} \in S$ and $b_{2}, \ldots, b_{n} \in B$ exist such that

$$
\begin{array}{ccc}
s_{1} & =s_{1} u_{1} & \\
s_{1} v_{1}=s_{1} u_{2} & u_{1} b=v_{1} b_{2} \\
\vdots & \vdots \\
s_{1} v_{n}=s_{1} & u_{n} b_{n}=v_{n} b_{1} .
\end{array}
$$


Then $a S \otimes B$.

Using Lemma 3.1 we obtain the following useful observation (see [8, Lemma 3]).

Lemma 3.2. Suppose $S$ is a monoid, $A$ is a right $S$-set and $B$ is a weakly flat left $S$-set. Assume $a, a^{\prime} \in A$ and $b, b^{\prime} \in B$ are such that $a \otimes b=a^{\prime} \otimes b^{\prime}$ in $A \otimes B$ via a scheme of length 1. Then $a \otimes b=a^{\prime} \otimes b^{\prime}$ in $\left(a S \cup a^{\prime} S\right) \otimes B$.

Proof. Suppose $a_{1} \in A, s_{1}, t_{1} \in S$ and

$$
\begin{aligned}
a & =a_{1} s_{1} \\
a_{1} t_{1} & =a^{\prime}
\end{aligned} \quad s_{1} b=t_{1} b^{\prime}
$$

is a scheme of length 1 yielding $a \otimes b=a^{\prime} \otimes b^{\prime}$ in $A \otimes B$. By Proposition 1.2 there exist $\alpha_{1}, \beta_{1} \in S$ and $b^{\prime \prime} \in B$ such that $s_{1} \alpha_{1}=t_{1} \beta_{1}$ and $s_{1} b=t_{1} b^{\prime}=s_{1} \alpha_{1} b^{\prime \prime}=t_{1} \beta_{1} b^{\prime \prime}$. By Lemma 3.1 it follows that $a \otimes b=a \otimes \alpha_{1} b^{\prime \prime}=a \alpha_{1} \otimes b^{\prime \prime}$ in $a S \otimes B$ and similarly $a^{\prime} \otimes b^{\prime}=a^{\prime} \beta_{1} \otimes b^{\prime \prime}$ in $a^{\prime} S \otimes B$. Furthermore $a \alpha_{1}=a_{1} s_{1} \alpha_{1}=a_{1} t_{1} \beta_{1}=\alpha^{\prime} \beta_{1} \quad$ and so $a \otimes b=a^{\prime} \otimes b^{\prime} \quad$ in $\left(a S \cup a^{\prime} S\right) \otimes B$.

Remark 3.3. Suppose $S$ is a monoid, $A$ is a right $S$-set, and $B$ is a weakly flat left $S$ set. Assume $a, a^{\prime} \in A$ and $b, b^{\prime} \in B$ are such that $a \otimes b=a^{\prime} \otimes b^{\prime}$ in $A \otimes B$ via a scheme

$$
\begin{array}{rlrl}
a & =a_{1} s_{1} & \\
a_{1} t_{1} & =a_{2} s_{2} & s_{1} b & =t_{1} b_{2} \\
& \vdots & \vdots \\
a_{i} t_{i} & =a_{i+1} s_{i+1} & s_{i} b_{i}=t_{i} b_{i+1} \\
& \vdots & \vdots \\
a_{n} t_{n} & =a^{\prime} & s_{n} b_{n}=t_{n} b^{\prime} .
\end{array}
$$

Our aim in the sequel will be to conclude, for particular monoids $S$, that $a \otimes b=a^{\prime} \otimes b^{\prime}$ in $\left(a S \cup a^{\prime} S\right) \otimes B$. This conclusion is valid if $n=1$ by Lemma 3.2. Suppose $n \geqq 2$ and that such a conclusion can be made for schemes of length $<n$. If $a_{i} t_{i}=a_{i+1} s_{i+1} \in a S \cup a^{\prime} S$ for some $i \epsilon\{1, \ldots, n-1\}$ then, using the inductive hypothesis twice, $a \otimes b=a_{i+1} s_{i+1} \otimes$ $b_{i+1}=a_{i} t_{i} \otimes b_{i+1}=a^{\prime} \otimes b^{\prime}$ in $\left(a S \cup a^{\prime} S\right) \otimes B$. In particular, if $t_{1} \in \mathrm{s}_{1} S$ or $s_{n} \in t_{n} S$ this will be the case. Moreover, if any of $s_{2}, \ldots, s_{n}, t_{1}, \ldots, t_{n-1}$ is equal to 1 , it is easy to see that the scheme may be replaced by one of shorter length, allowing the use of the inductive hypothesis.

Proposition 3.4. Let $S$ be a semigroup [with 0] which is the union of its [0-] minimal right ideals. Then every weakly flat left $S^{1}$-set is flat.

Proof. Suppose $A$ is a right $S^{1}$-set, $B$ is a weakly flat left $S^{1}$-set, $a, a^{\prime} \in A, b, b^{\prime} \in B$ and $a \otimes b=a^{\prime} \otimes b^{\prime}$ in $A \otimes B$ via the scheme 


$$
\begin{array}{rlrl}
a & =a_{1} s_{1} & & \\
a_{1} t_{1} & =a_{2} s_{2} & s_{1} b & =t_{1} b_{2} \\
& \vdots & \vdots \\
a_{n} t_{n} & =a^{\prime} & s_{n} b_{n}=t_{n} b^{\prime} .
\end{array}
$$

Following the discussion in Remark 3.3 we may assume $n \geqq 2$, and none of $s_{1}, t_{1}, s_{n}, t_{n}$ is 1 .

Suppose $S$ does not contain 0 . Note that in this situation $x S=x S^{1}$ for every $x \in S$. Since $s_{1} b=t_{1} b_{2}$ Proposition 1.2(ii) shows that $s_{1} S^{1} \cap t_{1} S^{1}=s_{1} S \cap t_{1} S$ is not empty. Thus $s_{1} S=t_{1} S, t_{1} \in S_{1} S^{1}$, and so Remark 3.3 furnishes the desired conclusion.

Now assume $S$ contains 0 . If $s_{1} S^{1} \cap t_{1} S^{1} \neq\{0\}$ then $t_{1} \in S_{1} S^{1}$ and the proof is completed as above. A similar argument applies if $S_{n} S^{1} \cap t_{n} S^{1} \neq\{0\}$. Suppose then that $s_{1} S^{1} \cap t_{1} S^{1}=\{0\}=s_{n} S^{1} \cap t_{n} S^{1}$. By Proposition 1.2(ii) there exist $\alpha_{1}, \beta_{1}, \alpha_{n}, \beta_{n} \in S^{1}$ and $\bar{b}, \hat{b} \in B$ such that $s_{1} \alpha_{1}=t_{1} \beta_{1}=0=s_{n} \alpha_{n}=t_{n} \beta_{n}, s_{1} b=t_{1} b_{2}=0 \bar{b}$ and $s_{n} b_{n}=t_{n} b^{\prime}=0 \hat{b}$. Because $a=a_{1} s_{1}$ and $s_{1} b=s_{1} \alpha_{1} b$ Lemma 3.1 yields $a \otimes b=a \otimes \alpha_{1} b$ in $a S^{1} \otimes B$. Similarly $a^{\prime} \otimes b^{\prime}=a^{\prime} \otimes \beta_{n} \hat{b}$ in $a^{\prime} S^{1} \otimes B$. From the scheme it is clear that $0 b=0 b^{\prime}$ and hence $\quad 0 \bar{b}=0 s_{1} b=0 b=0 b^{\prime}=O t_{n} b^{\prime}=0 \bar{b}$. Moreover $a \alpha_{1}=a_{1} s_{1} \alpha_{1}=a_{1} 0=a 0=a^{\prime} 0=a_{n} 0=$ $a_{n} t_{n} \beta_{n}=a^{\prime} \beta_{n}$. Therefore, in $\left(a S^{1} \cup a^{\prime} S^{1}\right) \otimes B, \quad a \otimes b=a \otimes \alpha_{1} \bar{b}=a \alpha_{1} \otimes \bar{b}=$ $a 0 \otimes \bar{b}=a \otimes 0 \bar{b}=a \otimes 0 \hat{b}=a 0 \otimes b^{\prime}=a^{\prime} \beta_{n} \otimes \hat{b}=a^{\prime} \otimes \beta_{n} \hat{b}=a^{\prime} \otimes b^{\prime}$.

Proposition 3.4 applies to [0-] simple semigroups containing a [0-] minimal right ideal and in particular to completely $[0-]$ simple semigroups and Croisot-Teissier semigroups (see [6, Chapter 8$]$ ).

We conclude with two partial results concerning the commutative situation.

Proposition 3.5. Suppose $S$ is a commutative monoid. Then every weakly flat cyclic $S$-set is flat.

Proof. Suppose $A$ and $B$ are $S$-sets with $B=S b$ cyclic, $a, a^{\prime} \in A$, and $a \otimes b=a^{\prime} \otimes b$ in $A \otimes S b$. We need only show that this equality holds in $\left(a S \cup a^{\prime} S\right) \otimes S b$. By assumption there exist $a_{1}, \ldots, a_{n} \in A, s_{1}, t_{1}, \ldots, s_{n}, t_{n} \in S$ such that

$$
\begin{array}{rlrl}
a & =a_{1} s_{1} & \\
a_{1} t_{1} & =a_{2} s_{2} & s_{1} b & =t_{1} b \\
& \vdots & \vdots & \\
a_{n} t_{n} & =a^{\prime} & s_{n} b & =t_{n} b .
\end{array}
$$

For each $i(1 \leqq i \leqq n)$ there exist $\alpha_{i}, \beta_{i} \in S$ such that $s_{i} \alpha_{i}=t_{i} \beta_{i}$ and $s_{i} b=t_{i} b=s_{i} \alpha_{i} b$ by Proposition 1.2. For convenience let $\beta_{0}=1, s_{n+1}=1$ and $a_{n+1}=a^{\prime}$. We use induction on $i$ to prove 
for each $i(1 \leqq i \leqq n), a_{i+1} s_{i+1} \beta_{1} \ldots \beta_{i} \in a S$ and $a_{i} s_{i} \beta_{1} \ldots \beta_{i-1} \otimes b=a_{i+1} s_{i+1} \beta_{1} \ldots \beta_{i} \otimes b$ in $a S \otimes S b$.

If $i=1$ then, since $a=a_{1} s_{1}$ and $s_{1} b=s_{1}\left(\alpha_{1} b\right)$, it follows from Lemma 3.1 that $a \otimes b=$ $a \otimes \alpha_{1} b$ in $a S \otimes S b$. Hence, $a_{1} s_{1} \beta_{0} \otimes b=a \otimes b=a \alpha_{1} \otimes b=a_{1} s_{1} \alpha_{1} \otimes b=a_{1} t_{1} \beta_{1} \otimes b=$ $a_{2} s_{2} \beta_{1} \otimes b$ in $a S \otimes S b$ as required. Now assume the result is true for a particular $i$ $(1 \leqq i \leqq n)$. Since $a_{i+1} s_{i+1} \beta_{1} \ldots \beta_{i}=a_{i+1}\left(s_{i+1} \beta_{1} \ldots \beta_{i}\right)$ and $\left(s_{i+1} \beta_{1} \ldots \beta_{i}\right) b=$ $\left(s_{i+1} \beta_{1} \ldots \beta_{i}\right)\left(\alpha_{i+1} b\right)$, it again follows from Lemma 3.1 that $a_{i+1} s_{i+1} \beta_{1} \ldots \beta_{i} \otimes b=$ $a_{i+1} s_{i+1} \beta_{1} \ldots \beta_{i} \otimes \alpha_{i+1} b$ in $a_{i+1} s_{i+1} \beta_{1} \ldots \beta_{i} S \otimes S b$, hence, in $a S \otimes S b$. Furthermore, $a_{i+1} s_{i+1} \beta_{1} \ldots \beta_{i} \alpha_{i+1}=a_{i+1} s_{i+1} \alpha_{i+1} \beta_{1} \ldots \beta_{i}=a_{i+1} t_{i+1} \beta_{i+1} \beta_{1} \ldots \beta_{i}=a_{i+2} s_{i+2} \beta_{i+1} \beta_{1} \ldots \beta_{i}$ and the conclusion follows easily.

Now, calculating in $a S \otimes S b, \quad a \otimes b=a_{2} s_{2} \beta_{1} \otimes b=\ldots=a_{n} s_{n} \beta_{1} \ldots \beta_{n-1} \otimes b=$ $a_{n+1} s_{n+1} \beta_{1} \ldots \beta_{n} \otimes b=a^{\prime} \beta_{1} \ldots \beta_{n} \otimes b . \quad$ Dually, $\quad a^{\prime} \otimes b=a \alpha_{1} \ldots \alpha_{n} \otimes b \quad$ in $\quad a^{\prime} S \otimes S b$. Moreover, $a \alpha_{1} \ldots \alpha_{n}=a_{1} s_{1} \alpha_{1} \ldots \alpha_{n}=a_{1} t_{1} \beta_{1} \alpha_{2} \ldots \alpha_{n}=a_{2} s_{2} \beta_{1} \alpha_{2} \ldots \alpha_{n}=a_{2} s_{2} \alpha_{2} \ldots \alpha_{n} \beta_{1}=\ldots=$ $a_{n} s_{n} \alpha_{n} \beta_{1} \ldots \beta_{n-1}=a_{n} t_{n} \beta_{n} \beta_{1} \ldots \beta_{n-1}=a^{\prime} \beta_{1} \ldots \beta_{n}$. Therefore $a \otimes b=a^{\prime} \otimes b$ in $\left(a S \cup a^{\prime} S\right) \otimes$ Sb.

Proposition 3.6. Let $S$ be a commutative monoid whose (principal) ideals form a chain. Then every weakly flat $S$-set is flat.

Proof. Suppose $A$ is an $S$-set, $B$ is a weakly flat $S$-set, $a, a^{\prime} \in A, b, b^{\prime} \in B$, and $a \otimes b=a^{\prime} \otimes b^{\prime}$ in $A \otimes B$. Then there exist $a_{1}, \ldots, a_{n} \in A, b_{2}, \ldots, b_{n} \in B, s_{1}, t_{1}, \ldots, s_{n}, t_{n} \in S$ such that

$$
\begin{aligned}
& a=a_{1} s_{1} \\
& a_{1} t_{1}=a_{2} s_{2} \quad s_{1} b=t_{1} b_{2}
\end{aligned}
$$

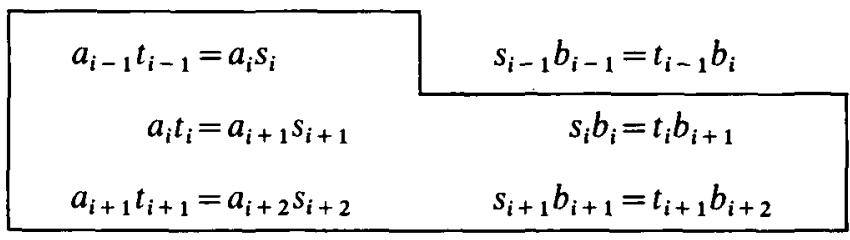

$$
\begin{aligned}
& a_{n} t_{n}=a^{\prime} \quad s_{n} b_{n}=t_{n} b^{\prime} .
\end{aligned}
$$

If $n=1, a \otimes b=a^{\prime} \otimes b^{\prime}$ in $\left(a S \cup a^{\prime} S\right) \otimes B$ by Remark 3.3. Now assume $n \geqq 2$ and $(\Sigma)$ is a scheme of minimum length joining $(a, b)$ to $\left(a^{\prime}, b^{\prime}\right)$ over $A$ and $B$. For convenience we let $a_{0}=a, a_{n+1}=a^{\prime}, b_{1}=b, b_{n+1}=b^{\prime}, t_{0}=1$, and $s_{n+1}=1$. Note that for $1 \leqq i \leqq n-1, s_{i} \in t_{i} S$ implies $t_{i} \in s_{i+1} S$, for otherwise, there exist $x, y \in S$ such that $s_{i}=t_{i} x$ and $s_{i+1}=t_{i} y$ (the ideals form a chain). But then the boxed portion of $(\Sigma)$ may be replaced by 


$$
\begin{aligned}
& a_{i-1} t_{i-1}=a_{i+1} s_{i+1} x \\
& a_{i+1} t_{i+1}=a_{i+2} s_{i+2} \quad s_{i+1} x b_{i}=t_{i+1} b_{i+2}
\end{aligned}
$$

contradicting the assumption that the length of $(\Sigma)$ is minimal. Similarly, for $1 \leqq i \leqq n-1$, $t_{i} \in s_{i+1} S$ implies $s_{i+1} \in t_{i+1} S$.

By Remark 3.3 we may assume $s_{1} \in t_{1} S$. From the observations above, $s_{1} \in t_{1} S$ implies $t_{1} \in s_{2} S$ implies $s_{2} \in t_{2} S$ implies ... implies $s_{n} \in t_{n} S$. Because $s_{n} \in t_{n} S$ the proof is complete, again using Remark 3.3.

\section{REFERENCES}

1. N. Bourbak!, Commutative Algebra (Addison-Wesley, Reading, Mass., 1972).

2. S. Bulman-Fleming and K. McDowell, Absolutely flat semigroups, Pacific J. Math. 107 (1983), 319-333.

3. S. Bulman-Fleming and $\mathrm{K}$. McDowell, Left absolutely flat generalized inverse semigroups, Proc. Amer. Math. Soc. 94 (1985), 553-561.

4. S. Bulman-Fleming and K. MCDowell, A characterization of left cancellative monoids by flatness properties, Semigroup Forum 40 (1990), 109-112.

5. S. Bulman-Fleming and K. McDowell, On V. Fleischer's characterization of absolutely flat monoids, Algebra Universalis 25 (1988), 394-399.

6. A. H. Clifford and G. B. Preston, The Algebraic Theory of Semigroups; vol. II (Mathematical Surveys of the American Math. Soc. 7, Providence, R.I., 1967).

7. J. M. Howie, An Introduction to Semigroup Theory (Academic Press, London, 1976).

8. V. Fleischer, Completely flat monoids, Učh. Zap. Tartu Un-ta 610 (1982), 38-52 (Russian). (MR85:20178). (English translation: Amer. Math. Soc. Transl. (2) 142 (1989), 19-31.)

9. J. Fountain, Right PP monoids with central idempotents, Semigroup Forum 13 (1977), 229-237.

10. M. KILP, On completely flat monoids, Tartu Riikl. Ūl. Toimetised 700 (1985), 32-37.

11. M. KILP, Strong flatness of flat cyclic left acts, Tartu Riikl. Ūl. Toimetised 700 (1985), $38-41$.

12. M. Kilp, Commutative monoids all of whose principal ideals are projective, Semigroup Forum 6 (1973), 334-339.

13. U. Knauer and M. Petrich, Characterization of monoids by torsion-free, flat, projective and free acts, Arch. Math. 36 (1981), 289-294.

14. P. Normak, On equalizer-flat and pullback-flat acts, Semigroup Forum 36 (1987), 293-313.

15. M. Y AMADA, Regular semigroups whose idempotents satisfy permutation identities, Pacific J. Math. 21 (1967), 371-392.

Department of Mathematics

WILFRID LaURIER UNIVERSITY

WaTERLoo, ONTARIO

CANADa, N2L 3C5 